\section{P-185 HELPING OUR STAFF FEEL VALUED AND RECOGNISED THROUGH POSITIVE FEEDBACK DURING THE COVID-19 PANDEMIC}

Katherine Fair, Cat Killin. Ayrshire Hospice, Ayr, UK

10.1136/spcare-2021-Hospice.201

In our hospice we work with an amazing group of colleagues who frequently go above and beyond for our patients. During the COVID-19 pandemic staff continued a fantastic level of patient-centred care, however, due to COVID-19 restrictions events such as parties and family gatherings couldn't be arranged. These events are some of the ways in which we make each day matter for our patients and we felt that staff often gained value from this. We wanted our staff to feel valued and recognised for small things, still possible despite COVID-19 restrictions, which they do everyday without thinking, that help to brighten a patient's day.

We designed a system to supply positive feedback to staff that was both COVID-19 safe and efficient. We designed a poster which could be laminated and distributed around the organisation with a QR code linking to a Google form. This could be scanned by any colleague and asked for the information to be fed back with an option of anonymity. An email was then sent from a 'positive feedback' mail box to the recipient with the positive feedback.

Over a four month period, over 100 messages of positive feedback have been sent out amongst colleagues. Overwhelmingly these have received a positive response and helped colleagues feel 'valued' and 'empowered'. Feedback forms have been submitted across a wide range of staff groups and have contained a huge range of feedback; from general words of encouragement to tales of amazing teachers, fantastic hairdressing and fixed glasses.

This project has been important for staff morale through difficult times. We hugely value the effort made every day by our colleagues and it is important to ensure our colleagues feel valued for the work that they do. We now plan to extend this project beyond the COVID-19 pandemic.

\section{P-186 'WELLNESS WEDNESDAY' - THE LITTLE THINGS THAT MAKE A BIG DIFFERENCE}

Natalia Carreja, Karen Garforth. Princess Alice Hospice, Esher, UK

\subsection{6/spcare-2021-Hospice.202}

Background The COVID-19 pandemic has had a significant effect on mental health (Pierce, Hope, Ford, et al., 2020; Chartered Institute of Personnel and Development, 2020). Workplace challenges like home-working, furlough, shielding, self-isolating and concerns about safety, meant that everybody was affected by the circumstances (Chandola, Kumari, Booker C, et al., 2020; White \& Van Der Boor, 2020). Early on, feelings of isolation, anxiety, worry, grief, uncertainty and stress were becoming the norm. This led to a recognition that paying attention to mental health and wellbeing was as important as the direct health consequences of the virus.

Aim A Wellbeing Survey was conducted in May 2020. In response to the feedback, and as part of a wider plan to support physical and mental wellbeing, we developed the following objectives:
- To keep in touch with staff regularly.

- To mitigate feelings of isolation and disengagement.

- To provide advice on how to look after oneself during challenging times.

Method A 'Wellness Wednesday' email was identified as a way of raising awareness of wellbeing. Our staff receive a weekly email about mental health and wellbeing-related subjects, including resilience, coping with change and uncertainty, and anxiety management. Online talks, webinars and events are also shared, along with raising awareness about diversity and inclusion. The email is written in a relaxed and upbeat tone to facilitate connections with staff.

Results A survey reflected that $91 \%$ of staff working from home, and $92 \%$ of staff working at the hospice valued email as the most useful form of communication. Feedback received about our 'Wellness Wednesday' emails reflects that staff find the support valuable and useful during these challenging times: 'Thank you, that's exactly what I needed to hear today!'

Conclusions Our 'Wellness Wednesday' email is an example of how small things can have a significant impact. We have identified this email as contributing to positive levels of staff engagement and we plan to continue to support our staff in this way going forward.

\section{P-187 NOT TRYING TO FIX YOU!}

Sarah Ireland. St Margaret's Hospice, Somerset, UK

\subsection{6/spcare-2021-Hospice.203}

Background As a result of the pandemic, the hospice's approach to supporting and engaging with staff and volunteers concerning resilience and wellbeing has evolved significantly over the past 18 months. There has been a clear need to be responsive, proactive, creative and engaging whilst encouraging staff and volunteers to take responsibility for developing their own resilience.

Aims

- To have regular engagement with staff (furloughed and not) to offer appropriate support.

- To be responsive to and anticipate the needs of the workforce, offering creative and wide-ranging options.

- To align with and support wellbeing initiatives across Somerset.

Methods Wide-ranging approaches from weekly webinars, virtual staff gatherings, taster and regular classes, learning opportunities, and a core resilience programme for managers, focusing on all aspects of resilience.

Results Staff resilience was measured initially by weekly 'temperature checks' and then the 'emotions thermometer' which was introduced as the length and impact of the pandemic continued. We then adapted the offer to match the need. Staff understanding of their needs developed from support to wellbeing and then into resilience.

Unintended consequences - greater take up for clinical supervision and new online clinical update and education sessions created, linking across departments and services.

Reduction in sickness or attrition. New starters commented on level of support on offer at their three-months reviews.

Adaptive learning - Not all sessions were well attended but we continued to run them to be visible before deciding to finish one activity and shift to another approach. 
Conclusion The ongoing programme continues to connect and engage with staff - mental health, wellbeing and resilience are part of everyone's conversations. Staff stay connected and reach out - different staff are able to engage with different activities. The offer continues to be collaborative, involving colleagues from spiritual care, fundraising, supportive care and $\mathrm{HR}$, which makes it more sustainable and relevant.

\section{P-188 BURNOUT IN THE UK CHILDREN'S HOSPICE WORKFORCE: FINDINGS FROM THE SWICH STUDY}

Andrew Papworth, Bryony Beresford, Lorna Fraser, Suzanne Mukherjee, Andre Bedendo, Jo Taylor. University of York, York, UK

\subsection{6/spcare-2021-Hospice.204}

In the UK, the children's hospice sector has become increasingly concerned about levels of work-related stress among its staff. This stems from increasing evidence that staff wellbeing is associated with the quality, cost and safety of patient care, and an acknowledgment of the important role of those working in children's hospices.

The SWiCH study, conducted by the Martin House Research Centre at the University of York, aims to increase understanding about the work-related stressors and rewards experienced by children's hospice care staff, and to identify the staff support systems and organisational practices that offer the most potential to enhance wellbeing at work. This paper will present preliminary findings from the study.

A national survey of UK children's hospice organisations and their care staff was administered between May and November 2020. A total of 583 staff from 32 children's hospices responded.

Initial analysis of the survey shows that 12 percent of respondents were suffering from moderate to severe burnout. Overall, respondents had an average burnout score (on the Copenhagen Burnout Inventory) of $32.5(n=583)$, which compares to an average score of 45.7 amongst a small sample (c.2.5\%) of NHS staff surveyed during the COVID-19 pandemic. UK children's hospice staff who responded to the survey had an average Job Satisfaction score of $6.9(n=414)$, and an average Core Engagement score of $7.5 \quad(n=415)$, the latter of which compares to the average of 7.0 for NHS staff (2020).

Further data analysis for the study will explore how these outcomes relate to the work-related stressors and rewards of working in a children's hospice, and what staff support systems and organisational practices have an impact on psychological wellbeing.

\section{P-189 'OFF TO WAR WHEN I DIDN'T SIGN UP!', SUPPORTING HOSPICE STAFF MENTAL HEALTH DURING A GLOBAL PANDEMIC}

Laura Shukla, Jenni Homewood. St Helena Hospice, Colchester, UK

10.1136/spcare-2021-Hospice.205

Wellbeing and Mental Health initiatives put in place during the pandemic.

The emotional and psychological pressures of working within hospice care are well documented, however, the addition of a global pandemic plunged hospice workforces into an unknown and unchartered way of working. Suddenly the switch from dealing with the everyday death and dying of patients, changed to having staff's own mortality, fear and anxiety thrust into reality. Unforeseen challenges within the workplace led to many staff experiencing emotions and anxieties that could not have been predicted.

The quick succession of events that changed everyday practice at the beginning of the pandemic gave rise to a number of initiatives implemented to support staff mental health and wellbeing. These have included:

- Wobble room - a designated safe place for staff to visit and relax in.

- Wobble room booklet for those working offsite.

- Gifts of meals and drinks from local companies.

- Mindfulness and relaxation.

- Self-help videos.

The number of Mental Health First Aiders and freedom to speak up guardians has been increased across the workforce. In addition to this, staff were given information and contact details for a multitude of both national and local support avenues such as the Samaritans, NHS, Here for you and MIND, enabling support virtually through websites, webinars, online training and apps. Within the hospice a focus on wellbeing was central to communications on the workplace intranet. This then resulted in the sign up of 15 new workplace health champions who will support ongoing activities throughout the year. The initiatives that have proved successful will continue to be offered and available to staff as we transition away from COVID-19 and maintain a focus on staff wellbeing within the organisation.

\section{P-190 DEVELOPING THE SKILLS OF HOSPICE STAFF TO PROVIDE CLINICAL SUPERVISION}

${ }^{1,2}$ Andy Ashley, ${ }^{1}$ Katherine Downey, 'Sarah Popplestone-Helm. ${ }^{1}$ St Richard's Hospice, Worcester, UK; ${ }^{2}$ Herefordshire and Worcestershire Health and Care NHS Trust, Worcestershire, UK

\subsection{6/spcare-2021-Hospice.206}

There is a strong national driver from the Care Quality Commission and the Nursing \& Midwifery Council around the provision of clinical supervision. This has long been recognised as vital in supporting staff in palliative care with the emotional labour of work (Goodrich, Harrison, Cornwell, et al., 2015) and helps develop a learning culture which improves patient safety and care (Francis, 2013). During the pandemic, where high levels of staff stress (Pastrana, De Lima, Pettus, et al., 2021) have led to moral injury and burnout, clinical supervision is all the more important.

With the development of a supervision policy, we recognised certain groups of hospice staff were not accessing clinical supervision regularly and it was mainly provided externally. The project, led by the education department, set out to utilise the skills and experience of existing staff to create additional supervisors and began by running lunchtime workshops to engage interest. Over half the 230 staff at the hospice were clinicians, of which 15 attended two days of training led by the head of family support and consultant clinical psychologist. It included theory, practice, 